\title{
Mental Health and the Health System in Bangladesh: Situation Analysis of a Neglected Domain
}

\author{
Anwar Islam ${ }^{1, ~ *, ~ T u h i n ~ B i s w a s ~}{ }^{2}$ \\ ${ }^{1}$ School of Health Policy \& Management, Faculty of Health, York University, Toronto, Canada \\ ${ }^{2}$ Department of Public Health, North south University, Dhaka, Bangladesh \\ Email address: \\ anwarhill@yahoo.com (A. Islam), aislam@yorku.ca (A. Islam), anwar.islam@icddrb.org (A. Islam)
}

\section{To cite this article:}

Anwar Islam, Tuhin Biswas. Mental Health and the Health System in Bangladesh: Situation Analysis of a Neglected Domain. American Journal of Psychiatry and Neuroscience. Vol. 3, No. 4, 2015, pp. 57-62. doi: 10.11648/j.ajpn.20150304.11

\begin{abstract}
Mental Health constitutes a major public health challenge undermining the social and economic development throughout much of the developing world. It is estimated that mental disorders account for 13\% of the global burden of disease (WHO 2008). However, in most developing countries mental health remains utterly neglected by the health system. In Bangladesh, for example, a meager $0.5 \%$ of the total health budget is allocated to mental health. On the other hand, as more than $65 \%$ of the total expenditure on health is out-of-pocket expenses, mental illness takes a heavy toll on the poor and the disadvantaged Based on a review of secondary data, the paper assesses the current situation of mental health in Bangladesh. The paper suggests that mental health care system in Bangladesh faces multifaceted challenges such as lack of public mental health facilities, scarcity of skilled workforce, inadequate financial resource allocation and social stigma. Bangladesh still does not have a comprehensive mental health policy to strengthen the entire health system. Clearly, the most crucial challenge is the absence of a dynamic and proactive stewardship able to design and enforce policies to further strengthen and enhance the overall mental health care. Such strong leadership could bring about meaningful and effective health sector reform, which will work more efficiently for the betterment of the health and social and emotional wellbeing of the people of Bangladesh, and would be built upon the values of equity and accountability.
\end{abstract}

Keywords: Mental Health, Health Care System, Situation Analysis

\section{Introduction}

According to the World Health Organization (WHO) health is "a state of complete physical, mental and social well-being and not merely the absence of disease"[1]. Mental health, therefore, is an integral part of human health and wellbeing. Mental disorders constitute a major public health challenge and account for $13 \%$ of the global burden of disease measured as disability adjusted life years [2]. Low and middle income countries have higher burden of mental disorders than economically developed countries [3, 4]. A large number of people around the world are suffering from psychiatric disorders. Mental illness constitutes four of the ten most common causes of worldwide burden of disease, yet it remains low on the agenda of policy makers, particularly in developing countries like Bangladesh. Unfortunately numerous organizations and NGOs that work on chronic non-communicable diseases in Bangladesh also largely ignore mental health. In Bangladesh the term chronic diseases remain largely limited to diabetes, cancer, cardiovascular diseases and hypertension. Needless to say, these diseases are growing problems for the health system in Bangladesh. As Bangladesh slowly but surely completes its demographic and epidemiological transition; it is quit natural to expect that chronic diseases like diabetes, cancer, cardio vascular diseases and hypertension will emerge as major killers replacing traditional communicable diseases [5]. However, despite being part of the chronic diseases, mental illness of various types seldom gets attention when the issue of demographic and epidemiological transition is discussed. Contrary to overall general perception, mental illness constitutes a serious threat to the national health. According to WHO, more than 450 million people in the world are suffering from neuro-psychiatric disorders and in Bangladesh there are 15 million people suffering from mental illnesses of various types [6]. In other words, almost 10 percent of the population is in need of mental health services.

In Bangladesh, data related to mental health is scarce. So is 
the case with preparedness of the health system in providing mental health care and services. This comprehensive review was conducted to better understand the prevailing situation and health system preparedness to address mental health issues in Bangladesh.

\section{Methodology}

The paper is based on a review of published and unpublished reports. Online search was conducted for primary research articles on prevalence of mental disorders covering both rural and urban areas of Bangladesh. Most of the studies were focused on prevalence and co-morbid conditions of mental illness. A few studies also focused on utilization pattern of mental health services. The study also reviewed NCD- related operational plans of the Ministry of
Health and Family Welfare as well as that of a few major NOGs and mental health service providers in Bangladesh. Two electronic databases (PubMed and BanglaJOL) were extensively searched for relevant data and information with especial focus on South- East Asia in general and on Bangladesh in particular. However, the search was limited to English language studies, articles, reports and other materials only. In searching the data bases, the following key words were used: "mental disorder", "chronic disease", "depression", "mood disorder", "health services", "mental health services", and "Bangladesh". Titles and/ or abstracts of selected articles were manually searched to identify materials relevant for inclusion in the study. Reference lists from these studies were also searched to identify additional relevant studies /information. An extensive grey literature search was also conducted by the authors.

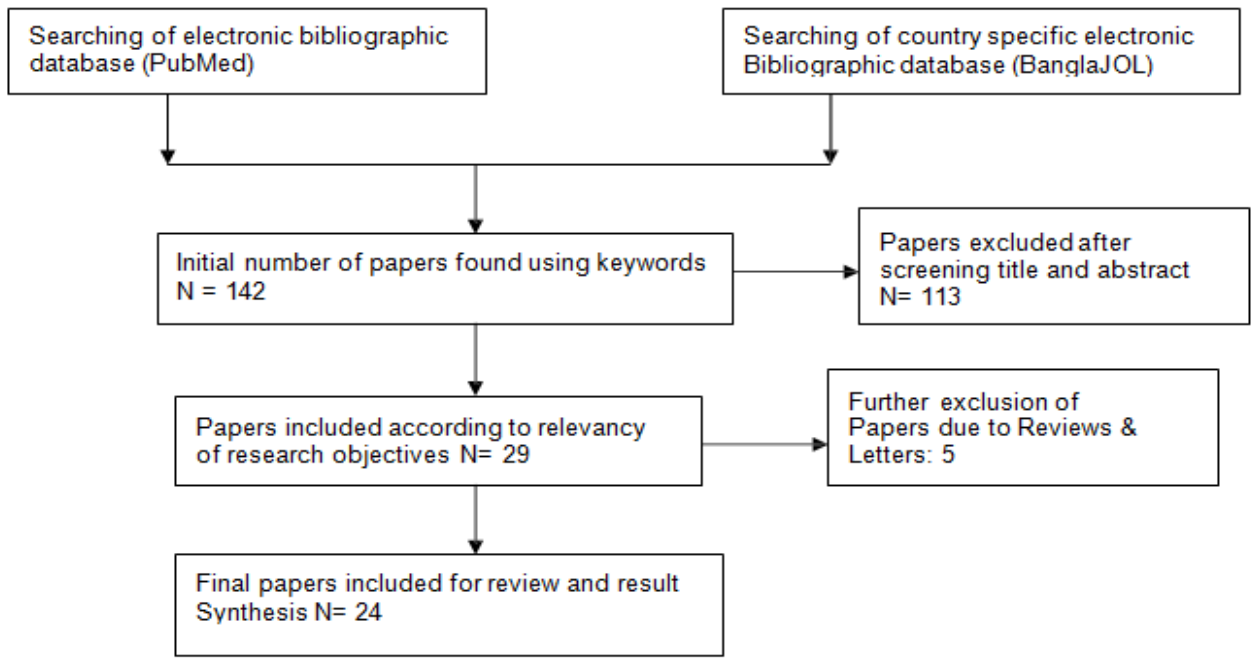

Figure 1. Data extraction process

\section{Results}

\subsection{Mental Health Situation in Bangladesh}

Figure 2 shows the prevalence of mental health disorders among the adult population in Bangladesh from 1974-2005 [7]. It is evident that although the prevalence of mental illness in Bangladesh declined significantly between 1974 (31.4\%) and $2005(16.1 \%)$, it was still alarmingly high in 2005. The first national survey on mental health conducted in 2003-2005 demonstrated that $16.1 \%$ of the adult population had some form of mental disorder and that the prevalence of mental disorders was higher among women (19\%) than men (12.9\%) [8]. In other words, in Bangladesh women are more vulnerable to mental illness than their male counterparts.

There is widespread stigma against people with mental illness in Bangladesh. There are many myths and superstitions surrounding the cause and outcome of mental illness. Mental disorders are primarily viewed as the result of being possessed by evil spirits rather than as illnesses that can be treated. Consequently, victims of mental illnesses are most often neglected, subjected to delayed care-seeking and abused [9].

Hossain et al in their systematic review study found that prevalence of mental disorders among children in Bangladesh ranged from $13.40 \%$ in 1998 to a high of $22.9 \%$ in 2004 (Figure-3). A community-based survey conducted in 2009 found the prevalence of mental disorders among children at $18.4 \%$ [10].

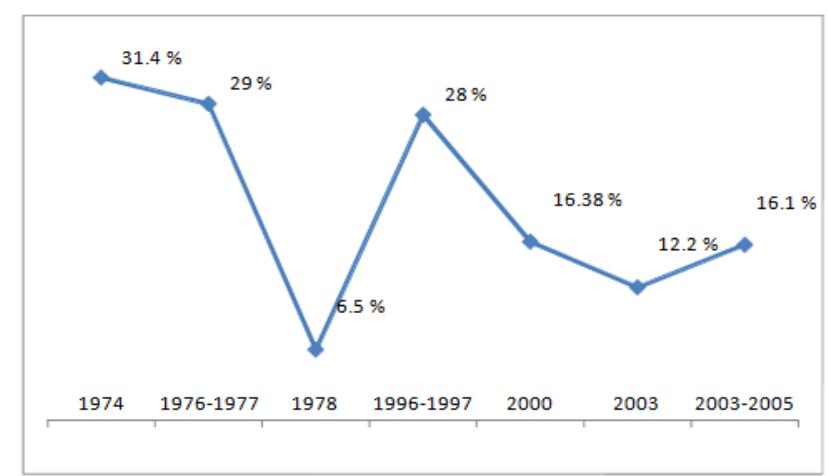

Source: Hossain et al 2014.

Figure 2. Prevalence of mental disorders among adults in both urban and rural areas. 


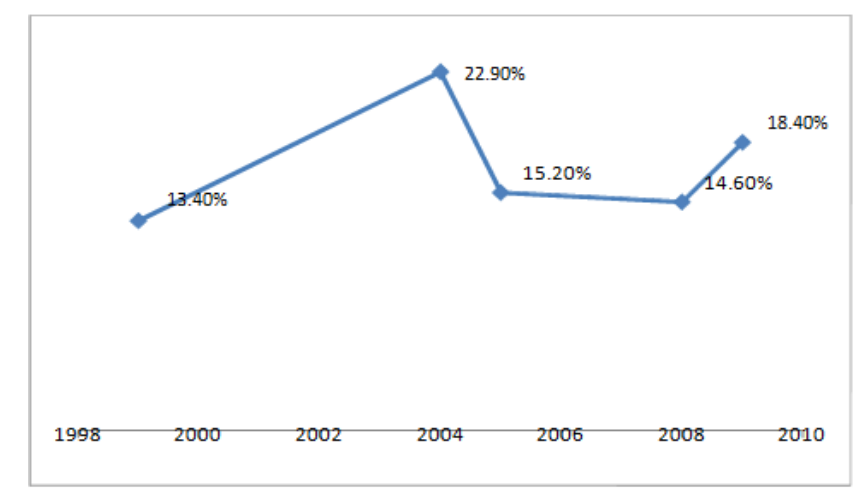

Source: Hossain et al 2014

Figure 3. Prevalence mental disorder among the children in Bangladesh.

\subsection{Diagnostic Distribution of Behavioral Disorders in Bangladesh}

The primary behavioral and mental illness-related diagnoses for women are depression and anxiety (Chowdhury et al., 1981). A recent study reports that among 28,998 deaths in women aged 10-50 years, 11.4 percent were attributable to suicide and depression (WHO, 2004). Priest et al. (2008) reported high prevalence of antenatal depression among rural women, who rarely seek treatment for their depression [9]. Another study revealed that 14 percent of women with depression admitted to thoughts of self-harm during their current pregnancy [10]. This study found that 33 percent of women suffered from depression, which is higher than the 12 percent prevalence reported in a systematic review of studies undertaken in developed countries [10]. Socio-cultural factors and physical violence were also found to be associated with antenatal depression in the study [11]. Researchers found that a woman who is subjected to torture by her husband has the highest probability of suffering from depression followed by an unhelpful or unsupportive mother-in-law or spouse. In most cases it is the preference for a male child and the failure to produce one by the pregnant woman that make the husband and/or the mother-in-law unhelpful and unsupportive [12] . Behavior disorders are also a common health concerns in Bangladesh. A study conducted by Rabbani and Hossain (1999) finds that 13.4 percent of children had behavior disorders (males 20.4 percent, females 9.9 percent) [13]. A two-phased survey carried out on over 10,000 children aged 2 to 9 revealed that the prevalence rates of severe and mild mental retardation were 0.6 percent and 1.4 percent respectively [14]. This survey also found that mild mental retardation was strongly and significantly associated with low socioeconomic status [14]. Durkin et al. (1993, 2000) used a structured measure for assessment of 162 flood affected children who had been diagnosed with behavioral disorders earlier; Durkin (2000) also found that an additional 10 percent had aggressive behavior and 34 percent had enuresis [15]. There is an increased probability of developing behavioral disorders for those who suffer from chronic illnesses such as diabetes, heart disease and asthma that equire timely treatment [16]. If people with chronic illnesses ignore their behavioral health problems, they are more likely to face negative health outcomes and substantial amount of additional money later for their severe illnesses [16]. Moreover, people with severe mental illness are also less likely to access general health care. A lack of proper diagnosis, inadequate and/or improper laboratory tests, inadequate training of behavioral health specialists, length of time spent without seeking care, inadequate space and equipment combined with stigma, fear, and shame hinder people with behavioral disorders from accessing mental health care [16].

\section{Discussion}

\subsection{Mental Health Services in Bangladesh}

Table-1 presents an overall picture of mental health services and their utilization in Bangladesh. It is clear that within the broader health sector, mental health has fewer facilities and beds. For example, beds in mental hospitals account for only $8 \%$ of the total number of hospital beds. On the other hand, there are no beds earmarked for the mentally ill in forensic units across the country. Consequently fewer mentally ill patients receive services in various institutional facilities. Only $26 \%$ of service users in outpatients facilities are mentally ill. Similarly, mentally ill constitute only $4.2 \%$ of the patients served in Inpatient Units.

\section{Table 1 Mental Health Facilities and their Utilization.}

\begin{tabular}{lc}
\hline Variables & $\%$ \\
\hline Beds in mental health facilities and other residential facilities \\
Mental Health Hospitals & $8 \%$ \\
Community Based Psychiatric & \\
Inpatients Units & $12 \%$ \\
Forensic Units & $0 \%$ \\
Community Residential facilities & $20 \%$ \\
Other Residential Facilities & $60 \%$ \\
Mentally Ill Patients treated in health facilities \\
Outpatients Facilities & $26.08 \%$ \\
Inpatients Units & $4.18 \%$ \\
Residential Facilities & $0.85 \%$ \\
Forensic Units & 0.00 \\
Percentages of female users treated in mental health facilities \\
Mental Hospitals & $19 \%$ \\
Residential Facilities & $81 \%$ \\
Inpatients Units & $42 \%$ \\
Outpatients Facilities & $44 \%$ \\
Percentage of children and adolescents & treated in mental health facilities \\
among all users & $0 \%$ \\
Mental Hospitals & $73 \%$ \\
Residential Facilities & $12 \%$ \\
Inpatients Units & $7 \%$ \\
Outpatients Facilities & \\
\hline & \\
\hline
\end{tabular}

Source: WHO-2007

The country also has a small number of community care 
facilities for patients with mental disorders. National Institute of Mental Health in Dhaka is the only national level tertiary care mental health treatment and research facility in the country [17]. Bangladesh has only one mental hospital that was established in 1957 (WHO, 2007). In 1969 the first outdoor clinic for the mentally ill was opened at the Dhaka Medical College in the capital Dhaka. Later in 1981, the National Institute of Mental Health (NIMH) was established to provide training on mental health to primary care physicians and health workers. In addition, a few NGOs are also involved in mental health in the country, primarily focusing on treatment and rehabilitation. In a country with 160 million people there are only a few outpatient mental health facilities $(n=50)$ and no community-based follow up care or day treatment facilities. As noted earlier, there is only one dedicated mental hospital in Bangladesh with 500 beds where the average length of stay of a patient is 137 days. The National Institute of Mental Health also runs a 150-bed hospital in Dhaka. There are also 15 beds in the forensic inpatient units, and 3,900 beds in residential facilities (e.g. homes for the destitute, inpatient detoxification centers, and homes for people with mental retardation). Quite a few substance abuse treatment and rehabilitation facilities are also there run by the private sector seldom mentioned in any official register. Most mental health facilities, unfortunately, are clustered in urban areas, especially in metropolitan cities. The absence of a specific mental health authority makes it difficult to systematically monitor and evaluate the mental health services in the country.

\subsection{Limited Mental Health Facilities}

A total of 536 public hospitals with 37,387 beds provide inpatient care services in Bangladesh for a population of 160 million [18]. In addition, there are 413 Upazila (sub-district) Health Complexes with limited inpatient care services [19]. There is only one 500-bed mental hospital in the country. There are 15 beds for mentally disordered people in forensic inpatient units and 3,900 beds in other residential facilities such as homes for persons with mental retardation, inpatient detoxification facilities, homes for the destitute, etc.

The density of psychiatric beds in or around Dhaka, the largest city, is 5 times greater than that in the rest of the country [20]. Antipsychotics, anxiolytics, anti-depressants, mood stabilizers and antiepileptic drugs are included in the list of essential medicines in the country [21]. However, psychotropic drugs are not widely available [22]. Few patients visiting government facilities are provided with psychotropic medicines.

\subsection{Financing of Mental Health Services}

Mental health expenditure from the Ministry of Health and Family Welfare is very insignificant and amounts to less than $0.5 \%$ of the total expenditure on health. Of all the expenditure on mental health, $67 \%$ are devoted to mental hospitals. In terms of affordability of mental health services less than $0.11 \%$ of the population has free access to essential psychotropic medicines. For those that pay out of pocket, the cost of antipsychotic medication using the cheapest available brand in local currency is Taka 5.00 (US\$ 0.07) per day and the cost of antidepressant medications using the cheapest available brand in local currency is Taka 3.00 (US\$ 0.04) per day. Social insurance schemes, rare as they are, do not cover any drugs for mental disorder.

\subsection{Human Resources for Mental Health}

Bangladesh has an acute shortage of human resources trained in psychosocial health and/or mental health. There are only 0.49 human resources trained and skilled in providing mental health services per 100,000-population. Sadly there are only 0.073 psychiatrists per 100,000 -population in the country most of them concentrated in major urban areas like the capital city of Dhaka. The breakdown of other types of human resources per 100,000-population is as follows: medical doctors (not specialized in psychiatry but working in mental health facilities) 0.18 ; nurses 0.19 ; psychologists 0.01 ; social workers 0.002 ; occupational therapists 0.002 ; and other mental health workers (including auxiliary staff, nonmedical primary health care workers, health assistants, medical assistants and professional and para-professional psychosocial counselors) 0.03 . Majority of the psychiatrists (54\%) work in both government administered mental health facilities and in private sector clinics; $46 \%$ work for NGOs/for-profit mental health facilities or in private practice [23]. It should be noted that due to such acute scarcity of trained human resources, psychiatrists working in government hospitals/facilities in Bangladesh are officially allowed to simultaneously work in the private sector. Sixtytwo percent of psycho-social professionals (psychologists, social workers, nurses and occupational therapists) work for government administered mental health facilities, $26 \%$ work for NGOs or in private practice, while $12 \%$ work for both the public and the private sectors.

As for psychiatrists - most scarce mental health human resource - 10 work in outpatient facilities, 33 in communitybased inpatient psychiatric units, and 4 in mental hospitals. On the other hand, 70 medical doctors not specialized in mental health, work in outpatient facilities; 140 in community-based psychiatric inpatient units and 15 in mental hospitals. As for nurses, 21 work in outpatient facilities, 220 in community-based psychiatric inpatient units and 140 in mental hospitals. Four psychosocial professionals (psychologists, social workers and occupational therapists) work in outpatient facilities; 3 in community-based psychiatric inpatient units and 3 in mental hospitals. Most of the other mental health workers (20) work in communitybased inpatient psychiatric units, and 10 in mental hospitals. It is unfortunate that none of these mental health workers choose outpatient facilities as their place of work. It is interesting to note that while there 0.04 psychiatrist per bed in community-based inpatient psychiatric units, in mental hospitals the psychiatrist-bed ratio is only 0.01. However, nurses are almost evenly distributed between the communitybased inpatient psychiatric unites $(0.27$ per bed $)$ and mental 
hospitals $(0.028$ per bed $)$. So is the case with other allied mental health professionals (psychologists, social workers, occupational therapists, etc). While in community- based inpatient psychiatric units there are 0.02 such allied professionals per bed, in mental hospitals there are 0.03 such allied workers per bed.

On the other hand, the distribution of human resources between urban and rural areas is grossly disproportionate. Most of the human resources (as well as tertiary level care facilities) are heavily concentrated in urban areas [18, $24,25]$. For example, the density of psychiatrists as well as psychiatric nurses in or around the largest city, the capital Dhaka, is 5 times greater than the density of psychiatrists and psychiatric nurses in the rest of the country.

\subsection{Mental Health Policy in Bangladesh}

The country has an old mental health policy named the Lunacy Act that was enacted and put in place in 1912 when the country was still a British colony (1757-1947). This policy reflects an outdated perception of mental illness and health. According to this policy, the term "lunatic" means "an idiot or a person with unsound mind". Besides defining the term, the policy also has a provision for asylum or prison for people with behavioral disorders if ordered by the courts. The Act also crafted a set of rules and norms to be followed by all actors involved with mental health. However, as these norms, rules and values were developed slightly more than 100 years ago, they and the Act embodying them need to be amended. India, a neighboring country of Bangladesh, has already amended the policy in 1987 by replacing the term "lunacy" to "mental illness," updating the relevant definitions, establishing new rules for licensing psychiatric hospitals, and developing procedures governing the practice of admitting and discharging people with mental illnesses [26]. Clearly Bangladesh is in critical need of a new Mental Health Act that would reflect the contemporary values and norms associated with mental illness. The people of Bangladesh in need of mental health care and services deserve quick action in replacing the outdated Lunacy Act of 1912.

In 2006 Bangladesh adopted a mental health policy, strategy and plan as part of its effort in promoting surveillance and prevention of Non-communicable Diseases (NCDs) [27]. The policy recommended a community-based approach in addressing all mental health issues including the provision of basic mental health services as part of the primary health care system (WHO and MOHFW, 2006). Nevertheless, mental health care in Bangladesh is still largely administered under the outdated Indian Lunacy Act of 1912. To remedy this situation, a new Mental Health Act has been drafted and is currently awaiting legislative approval.

\subsection{Social Stigma and Mental Health}

In Bangladesh the level of awareness of and medical care sought for mental illness is very low. Besides there is significant social stigma attached to mental illness that has severe impact on the health seeking behavior of people suffering from psychosocial or mental illness. Social stigma, on the one hand, prevents them from seeking care and, on the other, makes them silently suffer from social isolation and discrimination. Consequently, morbidity from psychiatric illnesses remains high and a seldom understood and/or recognized public health problem in Bangladesh. About $60 \%$ of ever married women of reproductive age in Bangladesh reported having experienced sexual and/or physical violence that remains largely ignored by the government and the power elites. Moreover, very little is known about violence against unmarried female adolescents [11]. There are many different forms of gender-based violence including: domestic, dowry-related acid attacks, rape, forced abortion, and trafficking for prostitution. Victims of all such forms of violence suffer from severe enduring psychological as well as psychosomatic illnesses [28].

A growing national concern, drug and substance abuse by women and children has increased over the years. The trend of drug consumption is high among the youth and teenagers, between the age of 15 and 30 years. In recent years, drug and substance abuse is fast increasing among young/adolescent females [29]. Needless to say, there are severe mental health consequences due to drug and substances abuse but the services available are very limited. There are few government, private sector or NGO-run facilities for drug and substance abuse victims. Moreover, these are primarily located in urban areas leaving rural people devoid of such services.

\section{Conclusion}

Clearly there is an urgent need to address social stigma associated with mental illness and further strengthen the availability of and accessibility to mental health as well as substance/drug abuse services across the country. At the same time, Bangladesh also lacks appropriately trained adequate number of human resources for mental health services. Despite the great efforts of the Dhaka-based National Institute of Mental Health, the overall health care system is yet to recognize and respond to the needs of the mentally ill. Contrary to popular belief, it must be fully recognized by policy makers and planners alike that mental illness constitutes a serious threat to national health. It is important to note that the WHO defines mental health as a "state of well- being in which people realize their potential, can cope with normal life stresses, can work productively and can contribute to their community". Last, but not the least, Bangladesh urgently needs a twenty-first century Mental Health Act that can serve the interests of the mental ill most efficiently and effectively upholding the principles of equity and fundamental human rights for all.

\section{Acknowledgments}

The author would like to acknowledge the valued support provided by York University, Toronto, Canada and Global Health Institute, North south University, Dhaka, Bangladesh in carrying out the study. 


\section{References}

[1] Burns J: A Southern Alliance for Research and Innovation in Mental Health: editorial. African journal of psychiatry 2009, 12(3):181-183, 185.

[2] Mathers C, Fat DM, Boerma J: The global burden of disease: 2004 update: World Health Organization; 2008.

[3] Bass JK, Bornemann TH, Burkey M, Chehil S, Chen L, Copeland JR, Eaton WW, Ganju V, Hayward E, Hock RS: A United Nations General Assembly Special Session for mental, neurological, and substance use disorders: the time has come. PLoS medicine 2012, 9(1):e1001159.

[4] Hock RS, Or F, Kolappa K, Burkey MD, Surkan PJ, Eaton WW: A new resolution for global mental health. The Lancet 2012, 379(9824):1367-1368.

[5] Kahn K, Collinson MA, Gómez-Olivé FX, Mokoena O, Twine R, Mee P, Afolabi SA, Clark BD, Kabudula CW, Khosa A: Profile: Agincourt health and socio-demographic surveillance system. International journal of epidemiology 2012, 41(4):988-1001.

[6] Hosman C, Jané Llopis E, Saxena S: Prevention of mental disorders: Effective interventions and policy options: Summary report: Geneva: World Health Organization; 2004.

[7] Hossain MD, Ahmed HU, Chowdhury WA, Niessen LW, Alam DS: Mental disorders in Bangladesh: a systematic review. BMC psychiatry 2014, 14(1):216.

[8] welfare WMoHaf: WHO-AIMS REPORT ON MENTAL HEALTH SYSTEM IN BANGLADESH. 2007.

[9] Adams A, Ahmed T, El Arifeen S, Evans T, Huda T, Reichenbach L: The Lancet Bangladesh Team Innovation for universal health coverage in Bangladesh: a call to action. Lancet 2013:62150-62159.

[10] Gausia K, Fisher C, Ali M, Oosthuizen J: Antenatal depression and suicidal ideation among rural Bangladeshi women: a community-based study. Archives of women's mental health 2009, 12(5):351-358.

[11] Naved RT, Rimi NA, Jahan S, Lindmark G: Paramedicconducted mental health counselling for abused women in rural Bangladesh: an evaluation from the perspective of participants. Journal of health, population, and nutrition 2009, 27(4):477.

[12] Peters D, Kayne R: Bangladesh health labor market study: Final report. Johns Hopkins Bloomberg School of Public Health 2003:72.

[13] Rabbani M, Hossain M: Behavior disorders in urban primary school children in Dhaka, Bangladesh. Public health 1999, 113(5):233-236.

[14] Islam N: Bangladesh national drug policy: an example for the Third World? Tropical doctor 1999, 29(2):78-80.

[15] Durkin M: The epidemiology of developmental disabilities in low-income countries. Mental retardation and developmental disabilities research reviews 2002, 8(3):206-211.

[16] Lopez M, Coleman -Beattie, B., Jahnke, L., \& Sanchez, K. : Connecting Body and Mind: A Resource Guide to Integrated Health Care in Texas and the United States . Retrieved from Hogg Foundation for Mental Health: Services, Research, and Policy \& Education 2008.

[17] Choudhury WA, Quraishi FA, Haque Z: Mental health and psychosocial aspects of disaster preparedness in Bangladesh. International Review of Psychiatry 2006, 18(6):529-535.

[18] Islam A, Biswas T: Health System in Bangladesh: Challenges and Opportunities. American Journal of Health Research 2014, 2(6):366-374.

[19] Khan MM, R Hotchkiss D, Dmytraczenko T, Zunaid Ahsan K: Use of a Balanced Scorecard in strengthening health systems in developing countries: an analysis based on nationally representative Bangladesh Health Facility Survey. The International journal of health planning and management 2013, 28(2):202-215.

[20] McMichael AJ: The urban environment and health in a world of increasing globalization: issues for developing countries. Bulletin of the World Health Organization 2000, 78(9):11171126.

[21] Baumann P, Hiemke C, Ulrich S, Eckermann G, Gaertner I, Gerlach M, Kuss H, Laux G, Muller- Oerlinghausen B, Rao M: The AGNP-TDM expert group consensus guidelines: therapeutic drug monitoring in psychiatry. Pharmacopsychiatry 2004, 37(6):243-265.

[22] Reich MR: Bangladesh pharmaceutical policy and politics. Health policy and planning 1994, 9(2):130-143.

[23] Barkat A, Maksud A: EngenderHealth, Dhaka. 2003.

[24] Islam A, Biswas T: Chronic Non-Communicable Diseases and the Healthcare System in Bangladesh: Current Status and Way Forward. Chronic Dis Int 2014, 1(2):6.

[25] Islam A, Biswas T: Health System Bottlenecks in Achieving Maternal and Child Health-Related Millennium Development Goals: Major Findings from District Level in Bangladesh.

[26] Rastogi P: Mental Health Act, 1987-An Analysis. Journal of Indian Academic and Forensic Medicine, 27(3), 1-4,2005.

[27] MOHAFW. DGHS: Strategic plan for surveillance and prevention of non-communicable diseases in Bangladesh 2007-2010.

[28] Hossain KT, Sumon M, Rashid S: Violence against Women: Nature, Causes and Dimensions in Contemporary Bangladesh. Bangladesh e-Journal of Sociology 2013, 10(1). 